\title{
High Power Time Domain fNIRS Device
}

\author{
Ileana Pirovano, ${ }^{1, *}$ Rebecca Re, ${ }^{1,2}$ Davide Contini, ${ }^{1}$ Alessandro Torricelli, ${ }^{1,2}$ Lorenzo Spinelli ${ }^{2}$ \\ ${ }^{1}$ Politecnico di Milano, Dipartimento di Fisica, Piazza Leonardo da Vinci 32, 20133 Milano, Italy \\ ${ }^{2}$ Istituto di Fotonica e Nanotecnologie, Consiglio Nazionale delle Ricerche, Piazza Leonardo da Vinci 32, 20133 Milano, Italy
}

*e-mail address: ileana.pirovano@polimi.it

Abstract: A new high power time domain near-infrared spectroscopy device has been developed aiming new investigations in the field of brain and deep organs oxidative metabolism monitoring. (C) 2020 The Author(s)

\section{Introduction}

Time domain functional near-infrared spectroscopy (TD fNIRS) is a non-invasive optical technique that allows investigating the oxygenation status over time, and consequently the functional activation, of different biological tissues, e.g. brain cortex and skeletal muscles. Injecting pulsed light in the spectral range $600-1100 \mathrm{~nm}$, it is possible to exploits the diffusive properties of biological tissues and collect the re-emitted photons after they underwent different absorption and scattering events. The photon distribution of time-of-flight (DTOF) is acquired and it can be fitted with the analytical solution of the diffusion equation to retrieve the absorption and reduce scattering coefficients $\left(\mu_{\mathrm{a}}\right.$ and $\left.\mu_{\mathrm{s}}{ }^{\prime}\right)$, specific for the tissue investigated in every sampling point [1] and it can be time-gated discriminating early and late arriving photons to enhance depth discrimination. In literature, different examples of both commercially and in-house built TD fNIRS instrumentations can be found [2,3]. They employ different laser sources and single photon sensitive detectors suitable to exploit the Time Correlated Single Photon Counting (TCSPC) technique to reconstruct the photons DTOF. Due to electronic and mechanical limits, most of them usually work at the sampling frequency of $1 \mathrm{~Hz}$. This is usually enough when the aim is to follow common variations of hemodynamic parameters of the tissue during a functional activation. Moreover, for safety reasons, these instruments make use of a limited power of the light injected into to the tissue, typically below $2 \mathrm{~mW} / \mathrm{mm}^{2}$, thus limiting the overall signal-to-noise ratio (SNR) and the quality of measurements. However, for particular applications (e.g. brain resting state oscillation monitoring or deep muscles/organs investigations), it would be necessary to increase the measurement sampling rate and the depth sensitivity still guaranteeing a high SNR. For this purpose, a technological improvement of the currently existing instrumentation is necessary.

\section{High power TD fNIRS device}

We propose here a new in-house built TD fNIRS device, developed exploiting commercially available high power laser sources and time multiplexing approach: pulses at two different wavelengths are injected at the same time in the same location without the switching time limitation of a mechanical optical switch. Moreover, for the detection lines, cooled hybrid photomultipliers tubes (PMT) have been chosen in order to guarantee a low dark count rate and a high SNR, even for short acquisition time.

\subsection{Device description}

The instrument is equipped with two high power pulsed diode lasers (LDH-P-C, PicoQuant GmbH, Berlin, Germany) operating respectively at $689 \mathrm{~nm}$ and $828 \mathrm{~nm}$, electronically driven at $80 \mathrm{MHz}$ (PDL-828-L "Sepia II", PicoQuant $\mathrm{GmbH}$ ). After electronically controlled variable attenuation stages (DDR-100, OZ Optics LTD., Ontario, Canada), an optical beam combiner is used to merge the two wavelength pulses into one injection optical fiber (glass step index, core/cladding: 600/660 $\mu \mathrm{m}$, Numerical Aperture (NA): 0.22, OZ Optics LTD.). Up to four optical fiber bundles (glass step index, inner diameter: $3 \mathrm{~mm}$, NA: 0.59, Loptek GmbH, Berlin, Germany) recollect the light after it has travelled inside the tissue. Four cooled hybrid photomultipliers tubes (PMA Hybrid 50, PicoQuant GmbH) are employed as detectors and TCSPC boards integrated into the system HydraHarp 400 (PicoQuant GmbH) that communicates with the PC though a USB interface. A schematic of the overall system and a picture of it are reported in Fig.1. The device is arranged in a 19' rack with four wheels and has a modular structure where, besides the already described components, an Interruptible Power Supply (UPS) and a de-coupling transformer (REOMED 1000BV, Reo Italia s.r.l., Italy) are embedded to guarantee the power supply and isolate the device from the external main power network in case of failure. 


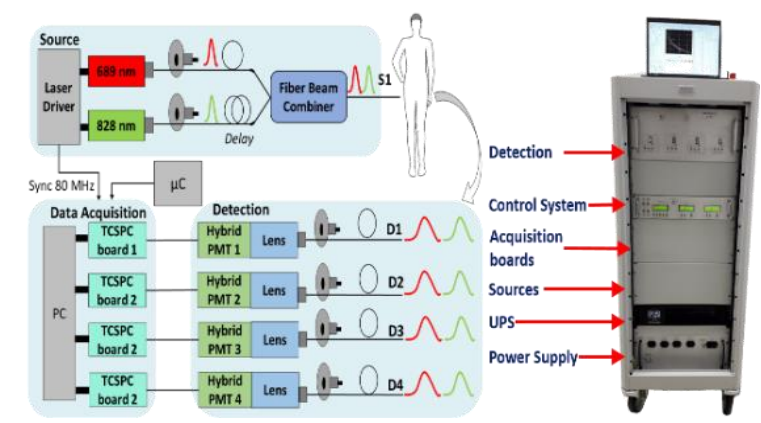

Fig. 1. Schematic representation of the high power TD fNIRS device developed and its picture.

\subsection{System characterization measurements}

In order to assess the performances of the TD fNIRS device built for further clinical applications, a series of characterization measurements have been carried out according to standardized protocols for diffuse optical spectroscopy instruments [4,5]. The instrument can deliver to the samples a maximum average power of $3 \mathrm{~mW}$ at $689 \mathrm{~nm}$ and $8.5 \mathrm{~mW}$ at $828 \mathrm{~nm}$ over a circular area with $7 \mathrm{~mm}$ diameter. The IRF has a Full Width at Half Maximum (FWHM) of about $400 \mathrm{ps}(350 \mathrm{ps})$ at $689 \mathrm{~nm}(830 \mathrm{~nm})$, while a warm-up time of 1 hour is needed in order to have stable features (i.e. counts per second, FWHM, peak temporal position) in the range of variability of $1 \%$. For what concerns the detectors performances, a responsivity of $2.97 \cdot 10^{-8} \mathrm{~m}^{2} \cdot \mathrm{sr}$ at $689 \mathrm{~nm}$ and $2.07 \cdot 10^{-8} \mathrm{~m}^{2} \cdot \mathrm{sr}$ at $828 \mathrm{~nm} \mathrm{has}$ been calculated. These values are comparable with results already reported for the same kind of detectors [4]. Moreover, the accuracy and the linearity of the instrument to retrieve the optical properties have been investigated over a set of calibrated solid phantoms. A source-detector distance of $2 \mathrm{~cm}$ was chosen for these measurements. In Fig. 2 a) the results obtained for phantoms with nominal $\mu_{\mathrm{a}}$ that ranges from $0.001 \mathrm{~cm}^{-1}$ to $0.49 \mathrm{~cm}^{-1}$ in eight steps and constant $\mu_{\mathrm{s}}$ ' equal to $10 \mathrm{~cm}^{-1}$ are reported. In Fig. 2b) the results refer to measurements where the nominal absorption coefficient was kept fixed at $0.07 \mathrm{~cm}^{-1}$ and nominal $\mu_{\mathrm{s}}$ 'varied from $5 \mathrm{~cm}^{-1}$ to $20 \mathrm{~cm}^{-1}$ in steps of $5 \mathrm{~cm}^{-1}$. A good linearity with varying the optical properties at both wavelengths can be observed, as well described by the linear interpolation performed over the data $\left(\mathrm{R}^{2}>0.99\right.$ in all cases). The percentage relative error for the estimated absolute values of $\mu_{\mathrm{a}}\left(\mu_{\mathrm{s}}{ }^{\prime}\right)$ is below $20 \%$ (40\%) with respect to the nominal values. A deviation form linearity and lower accuracy can be noticed for high values of absorption and low values of reduced scattering, since these conditions can not be well described by the diffusion approximation model used to retrieve the parameters.
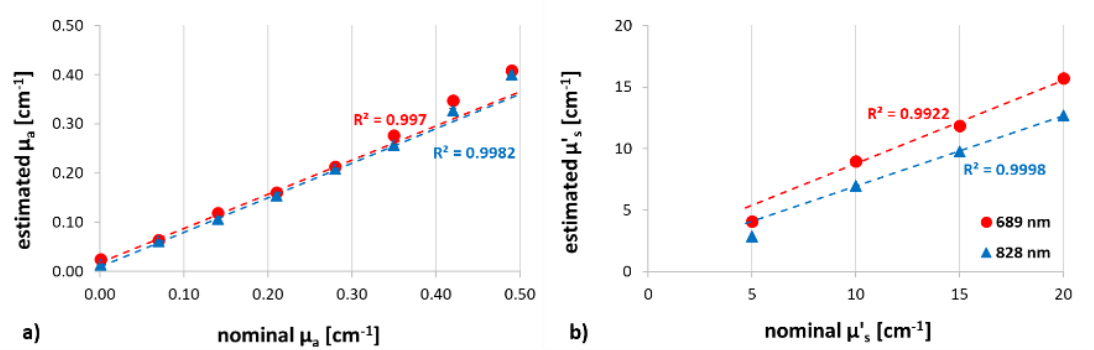

Fig. 2. Absorption and reduced scattering coefficients of calibrated phantoms estimated with the proposed device.

\section{Conclusions}

The new TD fNIRS instrument has been developed in the framework of the project "FHfFC - Future Home for Future Communities" funded by "III Accordo Quadro CNR-Regione Lombardia". The aim is to explore a new field of investigation where technological improvement are fundamental to guarantee good performances. Up to now, a characterization has been carried out to assess the basic performances of the instrument but a further investigation specific for high frequency measurement are going to be performed.

\section{References}

[1] A. Torricelli at al., "Time domain functional NIRS imaging for human brain mapping," Neuroimage 85, 28-50 (2014).

[2] F. Lange and I. Tachtsidis, "Clinical Brain Monitoring with Time Domain NIRS: A Review and Future Perspectives," Appl. Sci. 9, 1612 (2019).

[3] Y. Yamada et al., "Time-Domain Near-Infrared Spectroscopy and Imaging: A Review," Appl. Sci. 9, 1127 (2019).

[4] H. Wabnitz et al., "Performance assessment of time-domain optical brain imagers, part 1: basic instrumental performance protocol," J. Biomed. Opt. 19, 086010 (2014).

[5] A. Pifferi et al., "Performance assessment of photon migration instruments: the MEDPHOT protocol," Appl. Opt. 44, 2104-2114 (2005). 